\title{
Efecto del agua sobre el asfalto y su posible influencia en el daño por humedad en una mezcla asfáltica porosa
}

\author{
Effect of water on the bitumen and its possible influence in \\ the moisture damage on a porous asphalt mixture \\ Hugo Alexander Rondón Quintana ${ }^{1} \quad$ Juan Carlos Ruge Cárdenas ${ }^{2}$ \\ Luis Ángel Moreno Anselmi ${ }^{3 *}$ \\ Recibido 29 de agosto de 2014, aceptado 19 de enero de 2016 \\ Received: August 29, 2014 Accepted: January 19, 2016
}

\begin{abstract}
RESUMEN
Uno de los principales mecanismos de daño de mezclas asfálticas en servicio es el daño por humedad. Este fenómeno ocurre principalmente cuando se separa el ligante asfáltico del agregado pétreo debido a la presencia de agua (stripping). Diversos estudios han sido ejecutados con el fin de intentar entender el fenómeno. A pesar del alto número de investigaciones realizadas, las causas que lo generan no han sido totalmente entendidas e identificadas. El artículo presenta los resultados de un estudio experimental, ejecutado con el fin de evaluar la influencia que tiene el ligante asfáltico sobre el fenómeno, debido solo al efecto del agua. Sobre dos cementos asfálticos - CA (CA 80-100 y CA 60-70), se midió el cambio que experimentaron algunas de sus propiedades físicas (punto de ablandamiento - ASTM D36, viscosidad absoluta - ASTM D-2171, viscosidad a $135^{\circ} \mathrm{C}$ - AASHTO T-316 y penetración - ASTM D-5), químicas (ensayo SARA - ASTM D-4124) y reológicas (AASHTO T 315-05) cuando fueron sumergidos en agua durante 12 a 17 meses. Ensayos de resistencia bajo carga monotónica (Marshall, AASHTO T 245-97, 04) y de desgaste por abrasión (Cántabro, NLT 352/86) fueron ejecutados sobre una mezcla asfáltica porosa sumergida en agua durante 17 meses, y sobre la misma mezcla, pero fabricada con los asfaltos sumergidos en agua. Como conclusión general se reporta que los asfaltos en presencia de agua experimentan dentro de la mezcla contracción del ligante asfáltico, lo que podría ser una de las causas que generen el fenómeno de daño por humedad.
\end{abstract}

Palabras clave: Daño por humedad, mezcla asfáltica porosa, efecto del agua, Marshall, Cántabro.

\section{ABSTRACT}

One of the main forms of distress in asphalt mixtures is the moisture damage. This phenomenon occurs mainly when the asphalt binder is separated from the stone aggregate due to water presence. Many studies have been performed to describe and measure the moisture damage resistance in asphalt mixtures. Despite the high number of research projects performed, this phenomenon has not been fully understood yet. A laboratory study was initiated to investigate the influence of the asphalt binder on moisture damage, due to the effect of water only. Physical (softening point - ASTM D36, viscosity - ASTM D-2171, AASHTO

1 Facultad de Medio Ambiente y Recursos Naturales. Universidad Distrital Francisco José de Caldas. Avenida Circunvalar Venado de Oro. Bogotá D.C., Colombia. E-mail: harondonq@udistrital.edu.co

2 Facultad de Ingeniería Civil. Universidad Piloto de Colombia. Carrera 9 No 45A-44, 3er Piso, Edificio académico. Bogotá DC., Colombia. E-mail: juan-ruge@unipiloto.edu.co

3 Programa de Ingeniería Civil. Facultad de Estudios a Distancia. Universidad Militar Nueva Granada. Carrera 11 No 101-80, bloque D, oficina D 201. Bogotá DC, Colombia. E-mail: luis.moreno@unimilitar.edu.co

* Autor de correspondencia 
T-316 and penetration - ASTM D-5), chemical (SARA - ASTM D-4124) and rheological (AASHTO T 315-05) properties of two asphalt binders (AC 60-70 and AC 80-100) immersed in water during 12 to 17 months were evaluated. Mechanical characteristics of a porous asphalt mixture immersed in water, and manufactured with asphalt binders also immersed in water were evaluated using Marshall (AASHTO $T$ 245-97, 04) and Cantabro (NLT 352/86) tests. As concluding remark is reported that the interface asphalt binder-aggregate stone is affected for the change in the consistency that undergo the asphalt cements in presence of the water. Additionally, binders in presence of water undergo, inside the mixture, contraction, which may be one of the causes generating the stripping phenomenon.

Keywords: Moisture damage, porous asphalt mixture, water effect, Marshall, Cantabro.

\section{INTRODUCCIÓN}

\section{Antecedentes}

En una estructura de pavimento, uno de los principales mecanismos de deterioro de mezclas asfálticas en servicio es el daño por humedad [1-5]. En mezclas asfálticas este mecanismo de daño se refiere a la disminución de su resistencia mecánica y durabilidad debida principalmente a la pérdida de adherencia y cohesión por presencia de agua [6]. Este mecanismo de daño incide en la generación de otros modos de deterioro de mezclas asfálticas en servicio como son el agrietamiento por fatiga, agrietamientos tipo TDC (Top Down Cracking asociados a fenómenos térmicos, envejecimiento del ligante asfáltico, entre otros) y el ahullamiento o roderas. De acuerdo con [7], el agua genera pérdida de resistencia en la interface entre el ligante asfáltico y el agregado pétreo. Esto hace que la tasa de acumulación de la deformación permanente aumente debido a la pérdida de cohesión de la mezcla por humedad. De acuerdo con [8], este fenómeno fue identificado en la década de los treinta y la literatura de referencia lo denomina stripping [9-16]). [17] y [18] sugieren, que el stripping ocurre por reacción química entre el asfalto y el agregado. De manera similar, [10] y [19] mencionan, que la calidad del agregado y la composición mineralógica es uno, de entre tantos factores, que afectan la magnitud de este fenómeno [20]. Reportan, que la adherencia entre el ligante y el agregado pétreo es firmemente dependiente de la mineralogía de este último material [21]. Concluyen, que el fenómeno de stripping es mayor, directamente debajo de las ruedas de los vehículos [22] y [23]. Demostraron, que la edad del pavimento tiene una alta influencia en el daño por humedad [24]. Concluyen, que existe incompatibilidad térmica entre el agregado pétreo y el ligante, lo que genera contracción térmica diferencial de estos componentes, induciendo altos esfuerzos de tensión, grietas, deterioro localizado en la matriz de asfalto y deterioro de la interface ligante-agregado.

Las causas que generan el fenómeno de daño por humedad son complejas, ya que involucran aspectos físicos, químicos, mecánicos y termodinámicos [2527]. Mencionan adicionalmente que este fenómeno es función de la tensión superficial entre el agregado pétreo y el asfalto, y que el empleo de ligantes más viscosos genera mayor resistencia al daño por humedad. Además, sugieren que mezclas sometidas a ciclos de humedecimiento-secado o congelamientodeshielo, o agregados pétreos angulares pueden incrementar el potencial de stripping (este último debido a que las caras angulares son más difíciles de recubrir con asfalto). El estado del conocimiento en esta área es que, a pesar del alto número de investigaciones realizadas, el fenómeno de daño por humedad y las causas que lo generan no han sido totalmente entendidas e identificadas [28-31].

Para incrementar la resistencia al agua de mezclas en servicio son de amplia utilización los aditivos antistripping o mejoradores de adherencia [32-34]. Algunos aditivos son los tenso activos catiónicos, naftenato de hierro, cal hidratada, cemento Portland. Actualmente, incluso se están generando productos con nanotecnología que ayudan a repeler el agua dentro de la mezcla [5]. De acuerdo con [3], el daño por humedad de mezclas en servicio ocurre algunas veces debido a que en ocasiones prevalece el criterio de disminuir el contenido de asfalto en la mezcla para contrarrestar el fenómeno de ahuellamiento. Estos autores concluyen que, entre mayor sea el espesor de la película de asfalto adherida al agregado pétreo, mayor será la resistencia al daño por humedad. Una conclusión similar fue reportada por [35]. Por otro lado, entre mayor sea la adherencia 
y el recubrimiento del agregado pétreo fino con el asfalto (mastic), mayor será la durabilidad y resistencia al daño por humedad [36] [37]. Reporta que asfaltos más viscosos son menos propensos al daño por humedad y adicionalmente afirma, al igual que [38], que adiciones de cal mejoran la resistencia a este mecanismo de daño. Para intentar entender con mayor claridad el fenómeno, algunos investigadores proponen que el daño por humedad sea acoplado con un modelo de envejecimiento del asfalto o la mezcla [39], así como un modelo en donde se tenga en cuenta el paso de agua y aire por medio de las mezclas (permeabilidad) [40]. En síntesis, el daño por humedad debe ser acoplado a modelos en donde se tenga en cuenta la influencia del agua junto con otros elementos que conforman el medio ambiente como son el aire, la temperatura, los rayos ultravioleta, entre otros, para así poder evaluar la influencia de cada uno de ellos (desde el punto de vista mecánico y químico), de manera separada sobre el ligante y las mezclas [41]. Existen diversos ensayos para acondicionar muestras y medir la resistencia al daño por humedad de mezclas asfálticas. Algunos de ellos son: ensayo de inmersión estática (AASHTO T182), ensayo de agua hirviendo (ASTM D3625), método de la botella de balanceo (BSEN 12697-11), ensayo de tracción indirecta (AASHTO T283). En conclusión general se reporta que estas metodologías de ensayo tienen como limitación principal que no son capaces aún de simular con exactitud el mecanismo de daño por humedad de mezclas asfálticas [42].

Para poder entender este mecanismo de daño se hace necesario seguir descomponiendo las variables que inciden en su generación. Por lo anterior, en el presente estudio se desarrolló una metodología que busca ayudar a entender el efecto que tiene la interacción del agua y el asfalto sobre el mecanismo de daño por humedad de mezclas asfálticas.

\section{Síntesis}

Tradicionalmente se considera al asfalto como un material impermeable, cuya reacción ante agentes externos no es significativa. No obstante, como es conocido, el agua presenta elementos como oxígeno y compuestos que pueden originar cambios en las propiedades químicas y físicas del asfalto a largo plazo, principalmente cuando la interface ligantepétreo es delgada como ocurre en mezclas asfálticas. Por lo anterior, en el presente estudio se ejecutó una fase experimental tendiente a analizar la influencia que tiene el ligante asfáltico sobre el fenómeno de daño por humedad, evaluando tan solo el efecto del agua, es decir, no se tendrán en cuenta el efecto que puede causar el aire y los cambios de temperatura o la combinación aire-agua-temperatura. Para tal fin, láminas delgadas ( $2 \mathrm{~mm}$ de espesor) de cemento asfáltico (CA) tipo CA 80-100 y CA 60-70 fueron sumergidas en agua durante 17 meses $(80-100$ y 60-70 se refiere a un cemento asfáltico cuya medida en el ensayo de penetración ASTM D-5 está en un rango entre 80 y 100 décimas de $\mathrm{mm}$ y entre 60 y 70 décimas de $\mathrm{mm}$, respectivamente). Cada dos meses aproximadamente se retiraron del agua algunas láminas para fabricar mezclas asfálticas en caliente del tipo abiertas o porosas (MD de acuerdo con [43]), y se midieron sobre ellas, la resistencia que experimentaron bajo carga monotónica mediante el ensayo Marshall (AASHTO T 245-97, 04) y la resistencia por abrasión por medio del ensayo Cántabro (NLT 352/86). Estos resultados fueron comparados con aquellos que se obtuvieron sobre mezclas convencionales (fabricadas de la forma tradicional) sumergidas en agua. Se optó por utilizar mezclas abiertas en caliente debido a que, en un estudio anterior y similar, ya se evaluaron mezclas en caliente del tipo denso [44]. A las láminas de cemento asfáltico sumergidas se les realizaron ensayos de caracterización para medir si su consistencia y rigidez variaba cuando eran sumergidas en agua (Penetración-ASTM D-5, Punto de AblandamientoASTM D36, Ductilidad-ASTM D-113, Viscosidad Absoluta-ASTM D-2171, Viscosidad a $135{ }^{\circ} \mathrm{C}$ AASHTO T-316). Adicionalmente sobre ellas se ejecutaron ensayos para evaluar sus propiedades reológicas (AASHTO T 315-05) y químicas (ASTM D-4124), estas últimas basado en cromatografía líquida en columna para cuantificar la composición química de los asfaltos analizados mediante la determinación de las fracciones SARA (Saturados, Aromáticos, Resinas y Asfaltenos).

\section{TÉCNICAS EXPERIMENTALES}

\section{Caracterización de materiales}

En la Tabla 1 se presentan los valores obtenidos de los ensayos de caracterización ejecutados sobre el agregado pétreo. Los resultados de los ensayos de caracterización ejecutados sobre los cementos asfálticos CA 60-70 y CA 80-100 se presentan en la Tabla 2. Se observa que ambos materiales (agregado 
Tabla 1. Caracterización de los agregados.

\begin{tabular}{|l|l|c|}
\hline \multicolumn{1}{|c|}{ Test } & \multicolumn{1}{c|}{ Método } & Resultado (\%) \\
\hline Gravedad específica & ASTM D 854-00 & 2,62 \\
\hline Equivalente de arena & ASTM D 2419-95 & 76 \\
\hline Límite líquido & ASTM D 4318-00 & 0 \\
\hline Índice de plasticidad & ASTM D 4318-00 & 0 \\
\hline Caras fracturadas & ASTM D 5821-01 & 87 \\
\hline Índices de alargamiento y aplanamiento & NLT 354-91 & 9,5 \\
\hline Resistencia a sulfato de magnesio & ASTM C 88-99a & 12,9 \\
\hline Micro-Deval & ASTM D6928-03 & 22,3 \\
\hline $10 \%$ de finos (relación húmedo/seco) & DNER-ME 096-98 & 83 \\
\hline Ensayo máquina de Los Ángeles & ASTM C 131-01 & 24,6 \\
\hline
\end{tabular}

Tabla 2. Características generales de los asfaltos CA 80-100 y CA 60-70.

\begin{tabular}{|c|c|c|c|c|}
\hline Ensayo & Método & Unidad & $80-100$ & $60-70$ \\
\hline \multicolumn{5}{|c|}{ Ensayos sobre el asfalto original } \\
\hline Penetración & ASTM D-5 & $0,1 \mathrm{~mm}$ & 84 & 65 \\
\hline Punto de ablandamiento & ASTM D-36 & ${ }^{\circ} \mathrm{C}$ & 51,2 & 52,5 \\
\hline Viscosidad $\left(60^{\circ} \mathrm{C}\right)$ & ASTM D-2171 & Pa-s & 140,5 & 175,2 \\
\hline Viscosidad a $135^{\circ} \mathrm{C}$ & AASHTO T-316 & Pa-s & 0,35 & 0,36 \\
\hline Ductilidad & ASTM D-113 & $\mathrm{cm}$ & 139 & 117 \\
\hline Solubilidad en tricloroetileno & ASTM D-2042 & $\%$ & $>99$ & $>99$ \\
\hline Contenido de agua & ASTM D-95 & $\%$ & $<0,2$ & $<0,2$ \\
\hline Punto de inflamación & ASTM D-92 & ${ }^{\circ} \mathrm{C}$ & 295 & 275 \\
\hline \multicolumn{5}{|c|}{ Ensayos sobre el residuo luego del RTFOT } \\
\hline Pérdida de masa & ASTM D-2872 & $\%$ & 0,34 & 0,47 \\
\hline Penetración del residuo en $\%$ de la penetración original & ASTM D-5 & $\%$ & 62 & 72 \\
\hline
\end{tabular}

pétreo y asfaltos) presentan propiedades que los hacen aptos para la fabricación de mezclas asfálticas.

Adicionalmente se ejecutaron ensayos de caracterización reológica empleando un reómetro dinámico de corte (DSR por sus siglas en inglés, AASHTO T 315-05) (ver Tablas 3 y 4; T, F, G* y $\delta$ son la temperatura, frecuencia de carga, módulo de corte y ángulo de fase, respectivamente). El grado de desempeño es el mismo para ambos tipos de asfalto (CA 80-100 y CA 60-70), cuando se evalúa a altas e intermedias temperaturas de servicio: $58^{\circ} \mathrm{C}$ $\left(\mid \mathrm{G}^{*} / / \mathrm{sin} \delta>1.0 \mathrm{kPa}\right.$ para asfalto sin envejecer y $\left|\mathrm{G}^{*}\right| /$ $\sin \delta>2,2 \mathrm{kPa}$ para asfalto envejecido en RTFOT) y $22^{\circ} \mathrm{C}\left(\left|\mathrm{G}^{*}\right| \sin \delta<5000 \mathrm{kPa}\right.$ para asfalto envejecido en RTFOT+PAV), respectivamente (de acuerdo con la "AASHTO Performance Graded Binder Specification, MP1"). No se ejecutaron ensayos de caracterización reológica a bajas temperaturas de servicio ya que el país de origen donde se ejecutó el estudio es predominantemente tropical con algunas poblaciones ubicadas en zonas de clima templado.

\section{Diseño de mezclas asfálticas}

Luego de realizar los ensayos al agregado pétreo y a los cementos asfálticos se fabricaron cinco briquetas (compactadas a 50 golpes por cara y 1.200 $\mathrm{g}$ de masa) por cada porcentaje de asfalto de $3,5 \%$, $4,0 \%, 4,5 \%$ y $5,0 \%$, con el fin de realizar el ensayo Marshall (AASHTO T 245-97, 04). Adicionalmente para determinar el contenido óptimo de asfalto de la mezcla, se ejecutó el ensayo Cántabro (NLT 352/86). Para este ensayo se fabricaron también cinco briquetas (compactadas a 50 golpes por cara y $1.000 \mathrm{~g}$ de masa) por cada porcentaje de asfalto entre $3,5 \%$ y $5,0 \%$. Con el fin de verificar el cumplimiento de la granulometría requerida para fabricar mezclas MD, se alteró la granulometría original de los agregados, teniendo en cuenta como referencia los valores promedios en la franja 
Tabla 3. Caracterización reológica del CA 60-70.

\begin{tabular}{|c|c|c|c|c|c|}
\hline $\begin{array}{c}\mathbf{T} \\
{\left[{ }^{\mathbf{0}} \mathbf{C}\right]}\end{array}$ & $\begin{array}{c}\mathbf{F} \\
{[\mathbf{r a d} / \mathbf{s}]}\end{array}$ & $\begin{array}{c}\delta \\
{\left[{ }^{\mathbf{0}}\right]}\end{array}$ & $\begin{array}{c}\mathbf{G}^{*} \\
{[\mathbf{k P a}]}\end{array}$ & $\begin{array}{c}\left|G^{*}\right| / \sin \delta \\
{[\mathbf{k P a}]}\end{array}$ & $\begin{array}{c}\left|G^{*}\right| \cdot \sin \delta \\
{[\mathbf{k P a}]}\end{array}$ \\
\hline \multicolumn{7}{|c|}{ CA 60-70 sin envejecer } \\
\hline 58 & 10 & 87 & 2,47 & 2,47 & 2,47 \\
\hline 64 & 10 & 88 & 1,002 & 1,0 & 1,00 \\
\hline 70 & 10 & 89 & 0,453 & 0,45 & 0,45 \\
\hline \multicolumn{7}{|c|}{ CA 60-70 envejecido en RTFOT } \\
\hline 52 & 10 & 83 & 11,062 & 11,15 & 10,98 \\
\hline 58 & 10 & 85 & 4,276 & 4,29 & 4,26 \\
\hline 64 & 10 & 87 & 1,701 & 1,70 & 1,70 \\
\hline \multicolumn{7}{|c|}{ CA 60-70 envejecido en RTFOT + PAV } \\
\hline 16 & 10 & 44 & 14.266 & $20.536,7$ & 9.910 \\
\hline 19 & 10 & 45 & 10.193 & $14.415,1$ & 7.208 \\
\hline 22 & 10 & 47 & 6.659 & $9.105,0$ & 4.870 \\
\hline
\end{tabular}

Tabla 4. Caracterización reológica del CA 80-100.

\begin{tabular}{|c|c|c|c|c|c|}
\hline $\begin{array}{c}\mathbf{T} \\
\left.{ }^{\circ} \mathbf{C}\right]\end{array}$ & $\begin{array}{c}\mathbf{F} \\
{[\mathbf{r a d} / \mathbf{s}]}\end{array}$ & $\begin{array}{c}\delta \\
{\left[{ }^{\circ}\right]}\end{array}$ & $\begin{array}{c}\boldsymbol{G}^{*} \\
{[\mathbf{k P a}]}\end{array}$ & $\begin{array}{c}\left|G^{*}\right| / \sin \delta \\
{[\mathbf{k P a}]}\end{array}$ & $\begin{array}{c}\left|G^{*}\right| \cdot \sin \delta \\
{[\mathbf{k P a}]}\end{array}$ \\
\hline \multicolumn{6}{|c|}{ CA 80-100 sin envejecer } \\
\hline 58 & 10 & 87 & 1,980 & 1,98 & 1,98 \\
\hline 64 & 10 & 88 & 0,776 & 0,78 & 0,78 \\
\hline 70 & 10 & 89 & 0,413 & 0,41 & 0,41 \\
\hline \multicolumn{6}{|c|}{ CA 80-100 envejecido en RTFOT } \\
\hline 52 & 10 & 84 & 9,150 & 9,20 & 9,10 \\
\hline 58 & 10 & 85 & 2,698 & 2,71 & 2,69 \\
\hline 64 & 10 & 88 & 1,456 & 1,46 & 1,46 \\
\hline \multicolumn{6}{|c|}{ CA 80-100 envejecido en RTFOT + PAV } \\
\hline 16 & 10 & 47 & 11672 & $15.959,4$ & 8.536 \\
\hline 19 & 10 & 49 & 8534 & $11.307,6$ & 6.441 \\
\hline 22 & 10 & 52 & 5802 & $7.362,8$ & 4.572 \\
\hline
\end{tabular}

granulométrica (ver Tabla 5). Las temperaturas de compactación y de mezcla en el laboratorio fueron de $110^{\circ} \mathrm{C}$ y $120^{\circ} \mathrm{C}$, respectivamente. Estas temperaturas fueron escogidas teniendo en cuenta que para mezclas asfálticas porosas o abiertas la temperatura de fabricación que se recomienda por lo general es aquella donde el ligante alcance una viscosidad entre $700 \mathrm{cp}$ y $900 \mathrm{cp}$.

El porcentaje óptimo de cemento asfáltico escogido para la elaboración de las mezclas MD fue de 4,5\% para ambos tipos de CA. En este porcentaje: i) el desgaste por pérdida en abrasión en seco es inferior a $20 \%$ (ensayo Cántabro), ii) los vacíos con aire (AASHTO T 269) promedio de las mezclas MD fabricadas con CA 80-100 y CA 60-70 fueron de 22\% y $24 \%$, respectivamente, iii) la densidad volumétrica
Tabla 5. Granulometría mezclas tipo MD.

\begin{tabular}{|l|c|c|}
\hline \multicolumn{2}{|c|}{ Tamiz } & Porcentaje que pasa \\
\hline Normal & Alterno & MD \\
\hline $19,0 \mathrm{~mm}$ & $3 / 4 ”$ & 100 \\
\hline $12,5 \mathrm{~mm}$ & $1 / 2{ }^{\prime}$ & 85 \\
\hline $9,5 \mathrm{~mm}$ & $3 / 8^{\prime}$ & 62,5 \\
\hline $4,75 \mathrm{~mm}$ & $\mathrm{~N}^{\circ} 4$ & 23,5 \\
\hline $2,00 \mathrm{~mm}$ & $\mathrm{~N}^{\circ} 10$ & 14,5 \\
\hline $2,00 \mathrm{~mm}$ & $\mathrm{~N}^{\circ} 10$ & 14,5 \\
\hline $425 \mu \mathrm{m}$ & $\mathrm{N}^{\circ} 40$ & 8,5 \\
\hline $75 \mu \mathrm{m}$ & $\mathrm{N}^{\circ} 200$ & 5 \\
\hline
\end{tabular}

promedio de las mezclas MD fabricadas con CA $80-100$ y CA $60-70$ fue de $1,9 \mathrm{~g} / \mathrm{cm}^{3}$ y $1,86 \mathrm{~g} / \mathrm{cm}^{3}$, respectivamente, iv) la relación estabilidad-flujo (E/F) es muy cercano al mayor valor que experimentan las mezclas en el ensayo Marshall.

\section{Fases experimentales}

Con el fin de lograr los objetivos propuestos en el estudio el desarrollo experimental se dividió en tres fases:

Fase I. El objetivo de esta primera fase experimental fue medir la disminución típica que experimentan en su resistencia mecánica las mezclas cuando son sumergidas en agua. Para tal fin, inicialmente fueron fabricadas y sumergidas durante 16 a 17 meses, mezclas asfálticas tipo MD fabricadas con el contenido óptimo de asfalto $(4,5 \%)$ obtenido de la fase de diseño. La temperatura del agua oscilaba entre 15 y $20^{\circ} \mathrm{C}$ durante la noche y el día, respectivamente. Cada dos meses, durante los 17 meses de sumersión en agua fueron retiradas 20 muestras del agua para realizar sobre ellas ensayos Cántabro y Marshall (5 muestras por cada ensayo y tipo de asfalto). El agua utilizada era potable y provenía de la red de acueducto.

Fase II. En el mismo período de tiempo de la Fase I (16 a 17 meses), láminas de CA 80-100 y CA 60-70 con espesor de $2 \mathrm{~mm}$, apoyadas sobre una base de vidrio fueron sumergidas en agua. $\mathrm{Al}$ igual que en la fase anterior, la temperatura del agua oscilaba entre 15 y $20^{\circ} \mathrm{C}$. Cada dos meses aproximadamente eran retiradas láminas de asfalto del agua con el fin de fabricar 20 mezclas asfálticas tipo MD con el contenido óptimo de asfalto (4,5\%) (5 mezclas para cada ensayo y tipo de asfalto). Las mezclas 
elaboradas con estos ligantes fueron sometidas a los ensayos Marshall y al Cántabro. Este espesor de lámina fue el mínimo que se pudo fabricar sobre recipientes de vidrio de área rectangular $(30$ x 30 $\mathrm{cm}$ ) y espesor aproximado de $1 \mathrm{~cm}$. El objetivo de esta fase fue medir los parámetros mecánicos de los ensayos Cántabro y Marshall que desarrollaban las mezclas MD cuando estas eran fabricadas con los asfaltos sumergidos, los que habían cambiado sus propiedades por el efecto del agua.

Fase III. En esta fase se evaluó el efecto del agua sobre las propiedades de láminas de asfalto sumergidas con el fin de entender con mayor claridad los resultados obtenidos de las fases anteriores. Adicional a las láminas retiradas del agua en la Fase II para fabricar mezclas MD, en esta fase, otras láminas fueron retiradas con el fin de realizar ensayos de Penetración (ASTM D-5), Punto de Ablandamiento (ASTM D36), Viscosidad Absoluta (ASTM D-2171) y Viscosidad a $135^{\circ} \mathrm{C}$ (AASHTO T-316) para evaluar el cambio en la consistencia que experimentaban cuando eran sumergidas en agua. Antes de realizar los ensayos, la superficie de las láminas de asfalto era secada cuidadosamente con estopa. Luego el CA se separó de su soporte por medio de una espátula. Los ensayos de viscosidad absoluta $\left(60^{\circ} \mathrm{C}\right)$ y viscosidad a $135^{\circ} \mathrm{C}$ se realizaron utilizando un viscosímetro capilar de vacío y uno rotacional, respectivamente. Cada ensayo utilizó una muestra independiente, es decir, ninguna muestra de cemento asfáltico se reutilizó. Es importante resaltar, que en los ensayos mencionados con anterioridad, los asfaltos solo experimentan cambios en sus propiedades físicas debido a reacciones o trabajo químico por la interacción agua-asfalto. Por otro lado, se ejecutó una fase de caracterización reológica (AASHTO T 315-05) de las láminas de los asfaltos CA 80-100 y CA 60-70 sumergidos en agua en un período de tiempo $\mathrm{t}=12$ meses, que se comparó con los resultados obtenidos de los asfaltos sin sumergir $\mathrm{t}=0$ presentados en las Tablas 3 y 4 . Para evaluar la respuesta química que experimentaron los asfaltos sumergidos durante $t=12$ meses en agua fue realizado el ensayo de fraccionamiento SARA (ASTM D-4124), el cual consiste en la separación del ligante asfáltico en asfaltenos y maltenos por precipitación con $\mathrm{n}$-heptano y posterior fraccionamiento de los maltenos por medio de una columna de alúmina con solventes de polaridad creciente. Una descripción detallada de este ensayo puede ser consultada en [45]. El ensayo se ejecutó sobre una muestra sin sumergir $\mathrm{t}=0$ meses y sobre otra sumergida durante $\mathrm{t}=12$ meses.

\section{RESULTADOS}

Las Figuras 1-2 presentan los resultados de los ensayos Cántabro y Marshall, respectivamente, ejecutados sobre la mezcla porosa MD. Se observa para el caso de las mezclas sumergidas en agua (Fase I), una disminución notable en la resistencia al desgaste por abrasión en seco (Cántabro) y en la resistencia bajo carga monotónica (relación $E / F$ del ensayo Marshall). A los 16 meses de sumersión en agua, la pérdida por desgaste en seco aumentó en $182,4 \%$ y $194,5 \%$ para las mezclas fabricadas con CA 80-100 y CA 60-70, respectivamente, mientras que la relación $E / F$ en 17 meses de sumersión disminuyó $67 \%$.

Un comportamiento diferente experimentaron las mezclas que fueron fabricadas con láminas de CA sumergidas en agua (Fase II). Dichas mezclas desarrollaron un ligero incremento en la resistencia al desgaste por abrasión en seco y en la resistencia bajo carga monotónica. Se observa una disminución en la pérdida por desgaste en seco de aproximadamente $20 \%$, mientras que $E / F$ incrementó en $5,2 \%$ y $16,9 \%$ cuando las

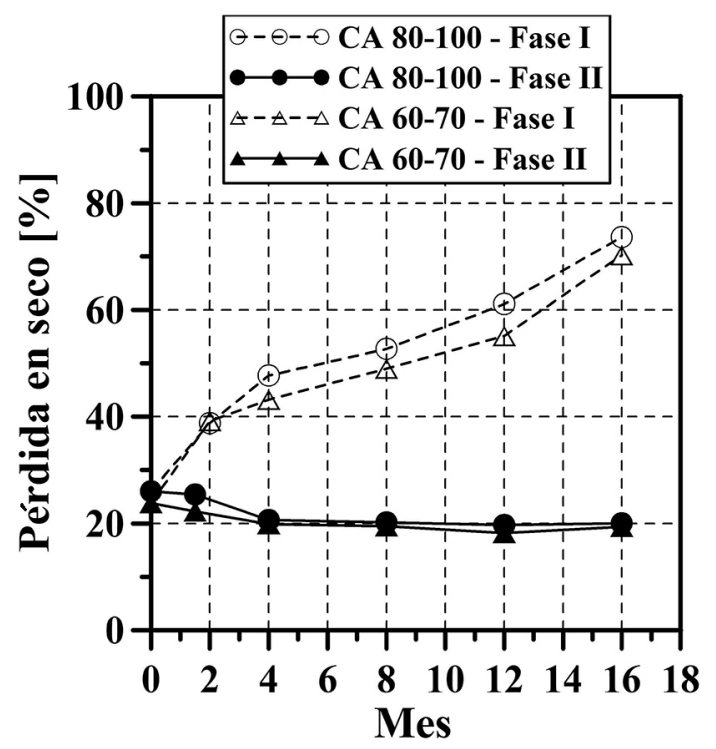

Figura 1. Resultados de evolución del ensayo Cántabro de la mezcla MD. 


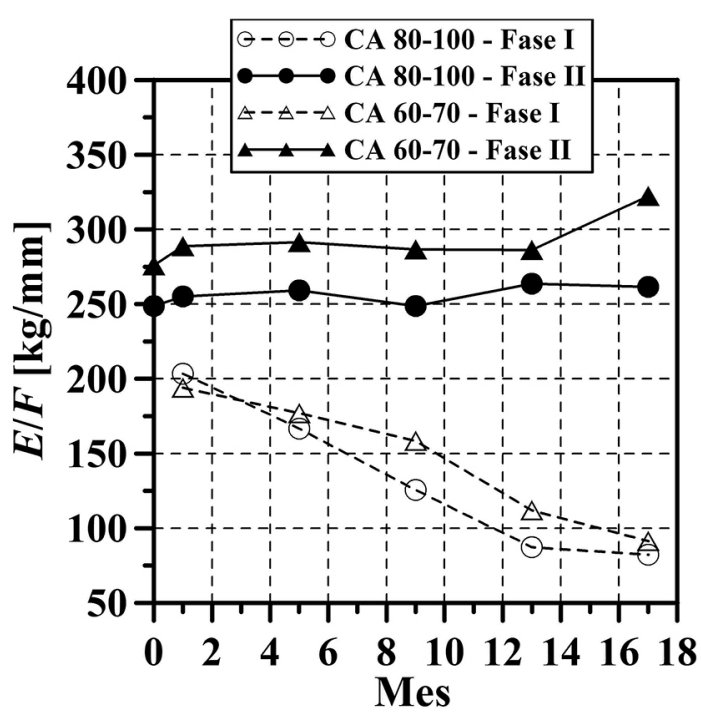

Figura 2. Resultados de evolución del ensayo Cántabro de la mezcla MD.

mezclas se fabricaron con CA 80-100 y CA 60-70, respectivamente. Este aumento en las resistencias mencionadas es debido principalmente al incremento en rigidez y viscosidad que experimentan las láminas de CA cuando son sumergidas en agua (Fase III, ver Figuras 3-5). A su vez, este aumento en rigidez es tal vez debido a un proceso de oxidación que experimenta la lámina de asfalto al contacto con el oxígeno del agua. En las Tablas 6 y 7 se presentan los resultados del ensayo de reología ejecutados sobre los asfaltos sumergidos en agua durante 12 meses. En comparación con los resultados

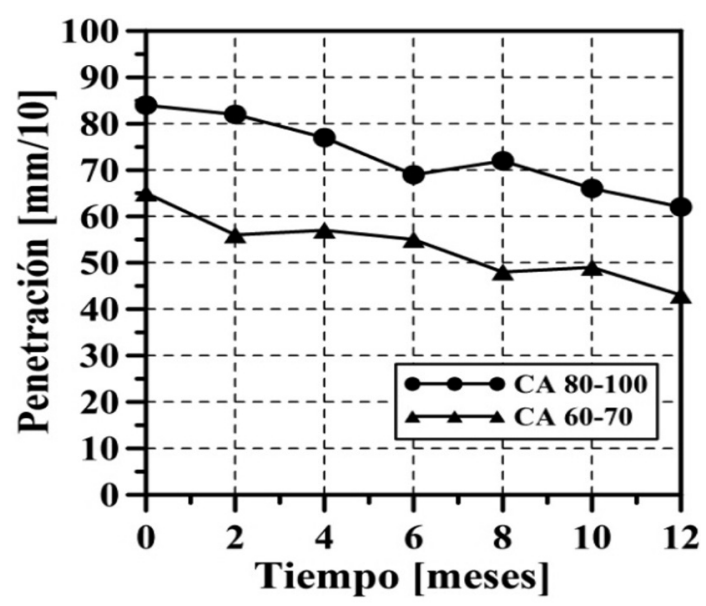

Figura 3. Evolución de la penetración de las láminas de CA con el tiempo de sumersión en agua.

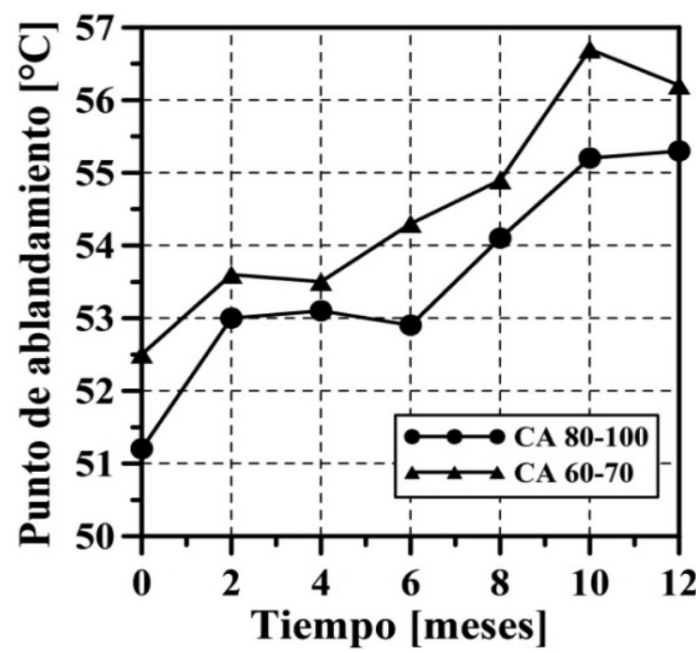

Figura 4. Evolución del punto de ablandamiento de las láminas de CA con el tiempo de sumersión.

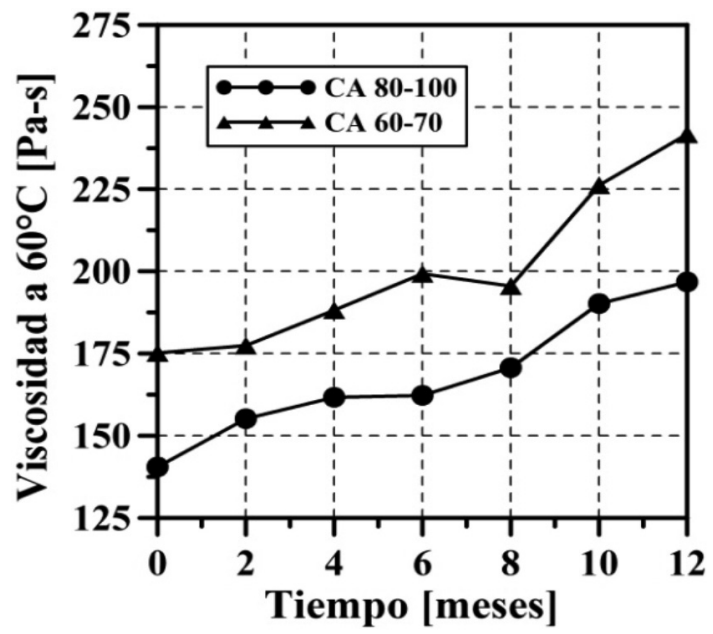

Figura 5. Evolución de la viscosidad absoluta $\left(60{ }^{\circ} \mathrm{C}\right)$ de las láminas de $\mathrm{CA}$ con el tiempo de sumersión en agua.

reportados en las Tablas 3 y 4 (asfaltos sin sumergir, $\mathrm{t}=0$ meses), los asfaltos CA 60-70 y CA 80-100 incrementaron su módulo de corte $\mathrm{G}^{*}$ entre $5 \%$ y $15 \%$ aproximadamente cuando las láminas fueron sumergidas en agua durante 12 meses. Adicionalmente se observa que la componente elástica de los asfaltos aumentó (disminución del ángulo de fase $\delta$ ), y esto hace prever que durante la interacción de los asfaltos con las moléculas de oxigeno del agua (oxidación), la respuesta elástica del asfalto se incrementa más rápidamente que la respuesta viscosa, debido al incremento 
en el tamaño de las moléculas por la presencia de oxígeno. Tal parece que las aglomeraciones moleculares pierden movilidad para fluir, el ángulo de fase decrece y se incrementa la viscosidad, lo que corresponde a cambios manifiestos en las propiedades reológicas [46-49].

En la Tabla 8, se presentan los resultados del ensayo SARA sobre los dos asfaltos sin sumergir $(t=0$ meses) y sumergidos en agua destilada durante $\mathrm{t}=12$ meses. Se reporta una disminución en los valores de las fracciones de los aromáticos (Ar), lo cual es característico del proceso de oxidación u envejecimiento de asfaltos, ya que actúan como

Tabla 6. Caracterización reológica del CA 60-70 después de sumersión en agua $\mathrm{t}=12$ meses.

\begin{tabular}{|c|c|c|c|c|c|}
\hline $\begin{array}{c}\mathbf{T} \\
{\left[{ }^{\mathbf{O}} \mathbf{C}\right]}\end{array}$ & $\begin{array}{c}\mathbf{F} \\
{[\mathbf{r a d} / \mathbf{s}]}\end{array}$ & $\begin{array}{c}\delta \\
{\left[{ }^{\circ}\right]}\end{array}$ & $\begin{array}{c}\boldsymbol{G}^{*} \\
{[\mathbf{k P a}]}\end{array}$ & $\begin{array}{c}\left|G^{*}\right| / \sin \delta \\
{[\mathbf{k P a}]}\end{array}$ & $\begin{array}{c}\left|G^{*}\right| \cdot \sin \delta \\
{[\mathbf{k P a}]}\end{array}$ \\
\hline \multicolumn{7}{|c|}{ CA 60-70 sin envejecer } \\
\hline 58 & 10 & 86 & 2,523 & 2,53 & 2,52 \\
\hline 64 & 10 & 87 & 1,24 & 1,24 & 1,24 \\
\hline 70 & 10 & 88 & 0,52 & 0,52 & 0,52 \\
\hline \multicolumn{6}{|c|}{ CA 60-70 envejecido en RTFOT } \\
\hline 52 & 10 & 81 & 12,27 & 12,42 & 12,11 \\
\hline 58 & 10 & 83 & 4,60 & 4,63 & 4,56 \\
\hline 64 & 10 & 86 & 1,99 & 1,99 & 1,98 \\
\hline \multicolumn{7}{|c|}{ CA 60-70 envejecido en RTFOT + PAV } \\
\hline 16 & 10 & 42 & 15.460 & $23.104,6$ & 10.345 \\
\hline 19 & 10 & 44 & 10.666 & $15.354,6$ & 7.409 \\
\hline 22 & 10 & 46 & 6.883 & $9.569,1$ & 4.952 \\
\hline
\end{tabular}

Tabla 7. Caracterización reológica del CA 80-100 después de sumersión en agua $\mathrm{t}=12$ meses.

\begin{tabular}{|c|c|c|c|c|c|}
\hline $\begin{array}{c}\mathbf{T} \\
{\left[{ }^{\mathbf{O}} \mathbf{C}\right]}\end{array}$ & $\begin{array}{c}\mathbf{F} \\
{[\mathbf{r a d} / \mathbf{s}]}\end{array}$ & $\begin{array}{c}\delta \\
{\left[{ }^{\circ}\right]}\end{array}$ & $\begin{array}{c}\boldsymbol{G}^{*} \\
{[\mathbf{k P a}]}\end{array}$ & $\begin{array}{c}\left|\boldsymbol{G}^{*}\right| / \mathbf{s i n} \delta \\
{[\mathbf{k P a}]}\end{array}$ & $\begin{array}{c}\left|G^{*}\right| \cdot \sin \delta \\
{[\mathbf{k P a}]}\end{array}$ \\
\hline \multicolumn{6}{|c|}{ CA 60-70 sin envejecer } \\
\hline 58 & 10 & 85 & 2,22 & 2,23 & 2,22 \\
\hline 64 & 10 & 86 & 0,89 & 0,89 & 0,89 \\
\hline 70 & 10 & 87 & 0,456 & 0,46 & 0,46 \\
\hline \multicolumn{6}{|c|}{ CA 60-70 envejecido en RTFOT } \\
\hline 52 & 10 & 82 & 10,48 & 10,58 & 10,38 \\
\hline 58 & 10 & 84 & 3,09 & 3,10 & 3,07 \\
\hline 64 & 10 & 87 & 1,68 & 1,68 & 1,68 \\
\hline \multicolumn{6}{|c|}{ CA 60-70 envejecido en RTFOT + PAV } \\
\hline 16 & 10 & 46 & 12.539 & $17.431,6$ & 9.020 \\
\hline 19 & 10 & 48 & 9.007 & $12.121,3$ & 6.694 \\
\hline 22 & 10 & 50 & 6.176 & $8.062,9$ & 4.732 \\
\hline
\end{tabular}

Tabla 8. Fracciones SARA correspondientes a los asfaltos analizados.

\begin{tabular}{|r|c|c|c|c|c|c|}
\hline \multicolumn{7}{|c|}{ CA 60-70 } \\
\hline Mes & $\begin{array}{c}\mathbf{S} \\
{[\%]}\end{array}$ & $\begin{array}{c}\mathbf{A r} \\
{[\%]}\end{array}$ & $\begin{array}{c}\mathbf{R} \\
{[\%]}\end{array}$ & $\begin{array}{c}\mathbf{A} \\
{[\%]}\end{array}$ & $\begin{array}{c}\text { IC } \\
{[\%]}\end{array}$ & $\begin{array}{c}\text { IS } \\
{[\%]}\end{array}$ \\
\hline 0 & 16,11 & 38,82 & 25,22 & 19,85 & 0,562 & 3,68 \\
\hline 12 & 15,79 & 37,33 & 24,18 & 22,70 & 0,626 & 3,43 \\
\hline \multicolumn{7}{|c|}{ CA 80-100 } \\
\hline Mes & $\begin{array}{c}\text { S } \\
{[\%]}\end{array}$ & $\begin{array}{c}\mathbf{A r} \\
{[\%]}\end{array}$ & $\begin{array}{c}\mathbf{R} \\
{[\%]}\end{array}$ & $\begin{array}{c}\mathbf{A} \\
{[\%]}\end{array}$ & $\begin{array}{c}\text { IC } \\
{[\%]}\end{array}$ & $\begin{array}{c}\text { IS } \\
{[\%]}\end{array}$ \\
\hline 0 & 16,71 & 43,54 & 28,92 & 10,83 & 0,380 & 5,28 \\
\hline 12 & 15,11 & 38,84 & 26,98 & 19,07 & 0,519 & 3,99 \\
\hline
\end{tabular}

medio de dispersión de los asfaltenos (A) [50-51]. El porcentaje de resinas (R), en ambos casos, también disminuye, dado que están constituidas por compuestos aromáticos polares susceptibles a la oxidación, dada su naturaleza estructural. Dos parámetros útiles para caracterizar el CA desde el punto de vista químico, mediante las cuatro fracciones mencionadas con anterioridad, son el índice de estabilidad coloidal (IC) y el índice de solubilidad (IS) ver ecuaciones (1) y (2). Por medio de estos índices es posible clasificar al ligante en tres estados: sol (ligante blando con pocos asfaltenos en comparación con los maltenos, típico de ligantes no envejecidos), sol-gel (estado apropiado del ligante en mezclas asfálticas) y gel (ligante rígido, tiene baja capacidad cohesiva y no garantiza la durabilidad de la mezcla asfáltica). IS $<4=$ gel, $4 \leq$ IS $\leq 9=$ sol-gel e IS $>9=$ sol. Para un buen desempeño del CA se recomienda que el $\mathrm{IC} \leq 0.6$, ya que si supera este valor el ligante experimenta una condición tipo gel.

$$
\begin{aligned}
& I C=\frac{S+A}{A r+R} \\
& I S=\frac{R}{A}+\frac{A r}{S}
\end{aligned}
$$

En la Tabla 9 se presenta la relación entre los aromáticos y los saturados $(A r / S)$, y las resinas y los asfaltenos $(R / A)$. Se observa que ambas relaciones disminuyen para los asfaltos analizados cuando son sumergidos durante 12 meses en agua. Esta disminución en las relaciones $R / A$ y $A / S$ son el factor característico del proceso asociado a la oxidación del asfalto [52-55]. 
Tabla 9. Evolución de los componentes químicos de los asfaltos sumergidos en agua destilada.

\begin{tabular}{|r|c|c|c|c|}
\hline \multirow{2}{*}{ Mes } & \multicolumn{2}{|c|}{ CA 60-70 } & \multicolumn{2}{c|}{ CA 80-100 } \\
\cline { 2 - 5 } & Ar/S & R/A & Ar/S & R/A \\
\hline 0 & 2,41 & 1,27 & 2,61 & 2,67 \\
\hline 12 & 2,36 & 1,07 & 2,57 & 1,41 \\
\hline
\end{tabular}

Los resultados reportados sugieren que el CA en presencia solamente de agua cambia sus propiedades ya que genera un aumento en la resistencia mecánica de las mezclas. Lo anterior conduce a pensar que la conexión ligante-pétreos en una mezcla asfáltica se puede romper (fenómeno de stripping) debido a que el CA, por la sola presencia del agua se rigidiza, lo que conlleva a una contracción del ligante y, por lo tanto, separación con el agregado. Una representación esquemática e idealizada del fenómeno se presenta en la Figura 6.

\section{CONCLUSIONES}

Un estudio experimental fue ejecutado con el fin de analizar la influencia que tiene el ligante asfáltico sobre el fenómeno de daño por humedad en mezclas asfálticas porosas, debido solo al efecto del agua. A pesar, que el cemento asfáltico es un material relativamente impermeable y de muy baja reacción química con agentes externos, como conclusión

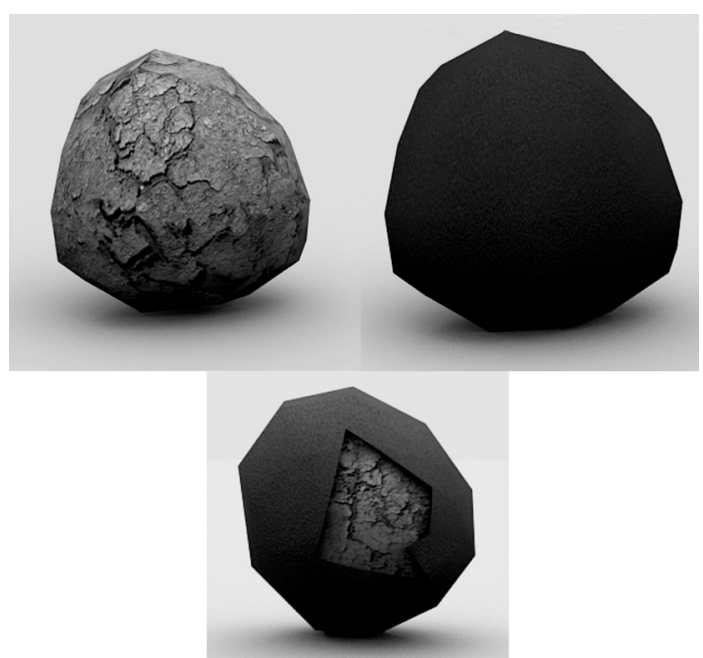

Figura 6. Esquema idealizado del proceso de contracción del CA y separación del agregado pétreo (stripping). general se reporta en este estudio, que este tipo de ligante en presencia única del agua cambia su consistencia (endurece) y aumenta su rigidez. Lo anterior podría ser debido, en parte, por la presencia de moléculas de oxígeno en el agua que reacciona con los cementos asfálticos (oxidación). Sin embargo, se deben ejecutar estudios a futuro que puedan validar esta hipótesis. Si esto fuere así, el envejecimiento y la oxidación entonces no se generarían solamente por la interacción aireasfalto o aire-agua-asfalto, como tradicionalmente se piensa, sino que adicionalmente el oxígeno presente en el agua podría contribuir a la generación de dicho fenómeno (interacción agua-asfalto). La rigidización y el endurecimiento que experimentaron las películas de asfalto sumergidas en agua se explican mrdiante los resultados de reología y del incremento de los índices coloidales en el ensayo SARA. Los resultados del fraccionamiento SARA para los asfaltos sumergidos en agua evidenciaron una disminución de la fracción de aromáticos con respecto al asfalto no sumergido, acompañado por un incremento de la fracción de los asfaltenos. Esto implica que la rigidización que experimentaron los asfaltos está asociada a un proceso que provoca el aumento en la polaridad de los asfaltos y una disminución en la fracción de aromáticos. Las relaciones aromáticos/saturados y resinas/asfaltenos disminuyeron para el caso de los asfaltos sumergidos en agua, lo que fue consistente con el aumento de los asfaltenos. La disminución de los compuestos aromáticos y de resinas puede ser responsable del endurecimiento observado en los asfaltos. Lo anterior demuestra que la interfase ligante asfáltico-agregado pétreo se afecta por este cambio de consistencia. El aumento reportado en la rigidez y viscosidad de los asfaltos en presencia de agua debe causar contracción del ligante asfáltico dentro de la mezcla, lo cual podría ser una de las causas que generen el desprendimiento del cemento asfáltico con el agregado pétreo (stripping). Adicionalmente se reporta que el asfalto CA 80-100 es más susceptible a cambiar sus propiedades físicas en presencia del agua en comparación con el CA 60-70.

\section{REFERENCIAS}

[1] M.R. Kakar, M.O. Hamzah and J. Valentin "A review on moisture damages of hot and warm mix asphalt and related investigations". 
Journal of Cleaner Production. Vol. 99, pp. 39-58. 2015.

[2] I.M.A. Abuawada, I.L. Al-Qadib and J.S. Trepanier. "Mitigation of moisture damage in asphalt concrete: Testing techniques and additives/modifiers effectiveness". Construction and Building Materials. Vol. 84, pp. 437-443. 2015.

[3] B. Sengöz and E. Agar. "Effect of asphalt film thickness on the moisture sensitivity characteristics of hot-mix asphalt". Building and Environment. Vol. 42, pp. 3621-3628. 2007.

[4] T.M. Breakah, J.P. Bausano, J.P. and R.Ch. Williams. "Integration of moisture sensitivity testing with gyratory mix design and mechanistic-empirical pavement design". Journal of Transportation Engineering. Vol. $135 \mathrm{~N}^{\circ}$ 11, pp. 852-857. 2009.

[5] F.M. Nejad, A.R. Azarhoosh, G.H. Hamedi and M.J. Azarhoosh. "Influence of using nonmaterial to reduce the moisture susceptibility of hot mix asphalt". Construction and Building Materials. Vol. 31, pp. 384-388. 2012.

[6] A.E.A.E. Behiry. "Laboratory evaluation of resistance to moisture damage in asphalt mixtures". Ain Shams Engineering Journal. Vol. $4 \mathrm{~N}^{\circ}$ 3, pp. 351-363. 2013.

[7] R.A. Tarefder, M. Zaman and K. Hobson. "A laboratory and statistical evaluation of factors affecting rutting". International Journal of Pavement Engineering. Vol. 4 $\mathrm{N}^{\circ}$ 1, pp. 59-68. 2003.

[8] S. Caro, E. Masad, A. Bhasin and D.N. Little. "Moisture susceptibility of asphalt mixtures, part 1: mechanisms". International J. of Pavement Engineering. Vol. $9 \mathrm{~N}^{\mathrm{o}} 2$, pp. 81-98. 2008.

[9] A. Aksoy, K. Samlioglu, S. Tayfur, and H. Ozen. "Effects of various additives on the moisture damage sensitivity of asphalt mixtures". Construction and Building Materials. Vol. 19, pp. 11-18. 2005.

[10] U. Bagampadde, U. Isacsson and B.M. Kiggundu. "Influence of aggregate chemical and mineralogical composition on stripping in bituminous mixtures". International J. of Pavement Engineering. Vol. $6 \mathrm{~N}^{\circ}$ 4, pp. 229239. 2005.

[11] R.B. Mallick, R. Pelland and F. Hugo. "Use of accelerated loading equipment for determination of long term moisture susceptibility of hot mix asphalt". International J. of Pavement Engineering, Vol. $6 \mathrm{~N}^{\mathrm{o}} 2$, pp. 125-136. 2005.

[12] S. Abo-Qudais. "The effects of damage evaluation techniques on the prediction of environmental damage in asphalt mixtures". Building and Environment. Vol. $42 \mathrm{~N}^{\circ} 1$, pp. 288-296. 2007.

[13] S. Abo-Qudais and H. Al-Shweily. "Effect of aggregate properties on asphalt mixtures stripping and creep behavior". Construction and Building Materials. Vol. 21, pp. 18861898. 2007.

[14] N. Kringos, T. Scarpas, C. Kasbergen, and P. Selvadurai. "Modelling of combined physical-mechanical moisture-induced damage in asphaltic mixes, part 1: governing processes and formulations". International J. of Pavement Engineering. Vol. $9 \mathrm{~N}^{\mathrm{o}} 2$, pp. 115-128. 2008.

[15] N. Kringos, T. Scarpas, A. Copeland and J. Youtcheff. "Modelling of combined physical-mechanical moisture-induced damage in asphaltic mixes, part 2: moisture susceptibility parameters". International J. of Pavement Engineering. Vol. $9 \mathrm{~N}^{\circ} 2$, pp. 129-151. 2008.

[16] E. Kassem, E. Masada, R. Lytton and R. Bulut. "Measurements of the moisture diffusion coefficient of asphalt mixtures and its relationship to mixture composition". International Journal of Pavement Engineering. Vol. $10 \mathrm{~N}^{\circ}$ 6, pp. 389-399. 2009.

[17] J.C. Peterson. "Chemistry of asphalt-aggregate interactions relationship with pavement moisture damage test". Transportation Research Record. N 83. Washington DC.: TRB, National Research Council, pp. 95-104. 1982.

[18] K.D. Stuart. "Evaluation of Procedure Used to Predict Moisture Damage in Asphalt Mixtures". Report No RD-8/091. Federal Highway Administration. 1986.

[19] A.K. Apeagyei, J.R.A. Grenfell and G.D. Airey. "Influence of aggregate absorption and diffusion properties on moisture damage in asphalt mixtures". Road Materials and Pavement Design. Vol. 16, Supplement 1, Special Issue: EATA 2015, pp. 404-422. 2015. 
[20] K. Kanitpong and H.U. Bahia. "Evaluation of HMA moisture damage in Wisconsin as it relates to pavement performance". International Journal of Pavement Engineering. Vol. 9 No 1, pp. 9-17. 2008.

[21] U. Bagampadde and B.M. Kiggundu. "Influence of truck load channelization on stripping in asphalt mixtures". Construction and Building Materials. $\mathrm{N}^{\circ} 21$, pp. 16281635. 2007.

[22] Q. Lu and J.T. Harvey. "Field investigation of factors associated with moisture damage in asphalt pavements". 10th International conference on asphalt pavements (ISAP), Quebec, Canada, pp. 691-700. 2006.

[23] Q. Lu, and J.T. Harvey. "Evaluation of moisture sensitivity of hot mix asphalt by flexural beam fatigue tests". Asphalt Concrete, ASCE, pp. 124-133. 2006.

[24] H.M. El Hussein, K.W. Kim and J. Ponniah. "Asphalt concrete damage associated with extreme low temperatures". Journal of Materials in Civil Engineering. Vol. $10 \mathrm{~N}^{\mathrm{o}} 4$, pp. 269-274. 1998.

[25] S. Abo-Qudais and H. Al-Shweily. "Effect of aggregate properties on asphalt mixtures stripping and creep behavior". Construction and Building Materials. $\mathrm{N}^{\circ} 21$, pp. 18861898. 2007.

[26] Abo-Qudais, S. and Al-Shweily, H. Effect of Antistripping Additives on Environmental Damage of Bituminous Mixtures. Building and Environment, Vol. 42, pp. 2929-2938, 2007.

[27] X. Cui, J. Zhang, N. Zhang, Y. Zhou, Z. Gao and W. Sui. "Laboratory simulation tests of effect of mechanical damage on moisture damage evolution in hot-mix asphalt pavement". International Journal of Pavement Engineering. Vol. $16 \mathrm{~N}^{\circ}$ 8, pp. 699-709. 2015.

[28] G.D. Airey, A.C. Collop, S.E. Zoorob and R.C. Elliott. "The influence of aggregate, filler and bitumen on asphalt mixture moisture damage". Construction and Building Materials. No 22, pp. 2015-2024. 2008.

[29] A. Varveri, J. Zhu and N. Kringos. "10 Moisture damage in asphaltic mixtures". Advances in Asphalt Materials - Road and Pavement Construction. A volume in Woodhead Publishing Series in Civil and Structural Engineering, pp. 303-344. 2015.
[30] M. Weldegiorgis and R. Tarefder. "Towards a Mechanistic Understanding of Moisture Damage in Asphalt Concrete". J. Mater. Civ. Eng. Vol. 27, N 3. 04014128. 2015.

[31] A. Mehrara and A. Khodaii. "A review of state of the art on stripping phenomenon in asphalt concrete". Construction and Building Materials. Vol. 38, pp. 423-442. 2013.

[32] H. Yao, Q. Dai and Z. You. "Chemo-physical analysis and molecular dynamics (MD) simulation of moisture susceptibility of nano hydrated lime modified asphalt mixtures". Construction and Building Materials. Vol. 101, Part 1, pp. 536-547. 2015.

[33] M. Nejad, F. Hamedi, and A. Azarhoosh. "Use of Surface Free Energy Method to Evaluate Effect of Hydrate Lime on Moisture Damage in Hot-Mix Asphalt". J. Mater. Civ. Eng. Vol. $25 \mathrm{~N}^{\circ}$ 8. 2013.

[34] B. Huang, X. Shu, Q. Dong and J. Shen. "Laboratory Evaluation of Moisture Susceptibility of Hot-Mix Asphalt Containing Cementitious Fillers". J. Mater. Civ. Eng. Vol. $22 \mathrm{~N}^{\circ}$ 7, pp. 667-673. 2010.

[35] K. Uppu, M. Hossain, L. Ingram and R. Kreider. "Moisture susceptibility of superpave mixtures with varying binder contents". Airfield and Highway Pavements, pp. 86-96. 2015. DOI: $10.1061 / 9780784479216.009$.

[36] M.I. Hossain and R.A. Tarefder. "Quantifying moisture damage at mastic-aggregate interface". International Journal of Pavement Engineering. Vol. $15 \mathrm{~N}^{\circ}$ 2, pp. 174-189. 2014.

[37] S.G. Jahromi. "Estimation of resistance to moisture destruction in asphalt mixtures". Construction and Building Materials. Vol. 23, pp. 2324-2331. 2009.

[38] S. Lee, S. Mun and Y.R. Kim. "Fatigue and rutting performance of lime-modified hotmix asphalt mixtures". Construction and Building Materials. Vol. 25, pp. 4202-4209. 2011.

[39] P.K. Das, H. Baaj, N. Kringos and S. Tighe. "Coupling of oxidative ageing and moisture damage in asphalt mixtures". Road Materials and Pavement Design. Vol. 16, Supplement 1, Special Issue: EATA 2015, pp. 265-279. 2015.

[40] R. Tarefder and M. Ahmad. "Evaluating the relationship between permeability and moisture damage of asphalt concrete 
pavements". J. Mater. Civ. Eng. Vol. $27 \mathrm{~N}^{\circ} 5$, 04014172. 2015.

[41] H.A. Rondón y F.A. Reyes. "PavimentosMateriales, Construcción y Diseño". Ed. ECOE, Bogotá D.C. 608 p. 2015.

[42] A. Diab and Z. You. "Development of a realistic conditioning and evaluation system to study moisture damage of asphalt materials". Airfield and Highway Pavement, pp. 10081017. 2013.

[43] INVIAS - Instituto Nacional de Vías. Especificaciones Generales para Construcción de Carreteras. Bogotá D.C., Colombia. 2013.

[44] H.A. Rondón y L.A. Moreno. "Influencia del agua en el fenómeno de stripping en mezclas asfálticas: estudio sobre el ligante". Revista Ingeniería y Universidad. Vol. 14 No 2, pp. 297-312. 2010.

[45] F.A. Reyes, C.E. Daza y H.A. Rondón, "Determinación de las fracciones SARA de asfaltos colombianos envejecidos al medio ambiente empleando Cromatografía Líquida en Columna". Revista EIA, Vol. $9 \mathrm{~N}^{\circ} 17$, pp. 47-56. 2012.

[46] J.C. Petersen. "A review of the fundamentals of asphalt oxidation". Transportation Research Circular. E-C140. 2009.

[47] L. Qi, Z.Q. Zhang, A.M. Sho and H.J. Yang. "Research on relationship between aging state and low-temperature performance of asphalt mixture". Journal of Testing and Evaluation. No 37, pp. 486-489. 2009.

[48] Y. Qi, F. Wang. "Study and evaluation of aging performance of petroleum asphalts and their constituents during oxygen absorption. I. Oxygen absorption behaviors and kinetics". Petroleum Science and Technology. Vol. 21 $\mathrm{N}^{\circ}$ 1, pp. 283-299. 2003.

[49] Y. Qi, F. Wang. "Study and evaluation of aging performance of petroleum asphalts and their constituents during oxygen absorption. II. Chemical group composition and structure changes". Petroleum Science and Technology. Vol. $22 \mathrm{~N}^{\circ} 3$, pp. 263-274. 2004.

[50] C.J. Glover, "New look for asphalt aging device". Texas Transportation Researcher (Texas Transportation Institute). Vol. 39 No 1,15 p. 2003.

[51] C.J. Glover, R.R. Davison and N. Vassiliev, "A new method for simulating hot-mix plant asphalt aging”. Project Summary Report 1742-S Project 0-1742: Improved HMAC Plant Binder Aging Simulation. Texas Transportation Institute, the Texas A\&M University System. 2003.

[52] J.P. Pfeiffer and R.N.J. Saal, "Asphaltic bitumen as colloidal system". The Journal of Physical Chemistry. N ${ }^{\circ} 43$, pp. 139149. 1940.

[53] J.C. Petersen, R.E. Robertson, J.F. Branthaver, P.M. Harnsberger, J.J. Duvall, S.S. Kim, D.A. Anderson, D.W. Christiansen and H.U. Bahia, "Binder Characterization and Evaluation". Vol. 1. SHRP A-367. Strategic Highway Research Program, National Research Council, Washington, DC. 152 p. 1994.

[54] J.C. Petersen, R.E. Robertson, J.F. Branthaver, P.M. Harnsberger, J.J. Duvall, S.S. Kim, D.A. Anderson, D.W. Christiansen, H.U. Bahia, R. Dongre, C.E. Antle, M.G. Sharma, J.W. Button and C.J. Glover, "Binder Characterization and Evaluation". Vol. 4: Test methods. SHRP A-370. Strategic Highway Research Program, National Research Council, Washington, DC. 1994.

[55] R.E. Robertson, "Properties of Asphalts and their Relationship to Pavement Performance". SHRP-A/UWP-91. Strategic Highway Research Program, National Research Council, Washington, DC. 1991. 